\title{
SELF-AFFINE SETS IN ANALYTIC CURVES AND ALGEBRAIC SURFACES
}

\author{
De-Jun Feng and Antti Käenmäki \\ The Chinese University of Hong Kong, Department of Mathematics \\ Shatin, Hong Kong; djfeng@math.cuhk.edu.hk \\ University of Jyväskylä, Department of Mathematics and Statistics \\ P.O. Box 35 (MaD), FI-40014 University of Jyväskylä, Finland; antti.kaenmaki@jyu.fi
}

\begin{abstract}
We characterize analytic curves that contain non-trivial self-affine sets. We also prove that compact algebraic surfaces do not contain non-trivial self-affine sets.
\end{abstract}

\section{Introduction}

Self-similar and self-affine sets are among the most typical and important fractal objects; see e.g. [2]. They can be generated by the so-called iterated function systems; see Section 2. Although these sets can be very irregular as one expects, they often have very rigid geometric structure.

It is not surprising that typical non-flat smooth manifolds do not contain any non-trivial self-similar or self-affine set. For instance, circles are such examples. To see this, suppose to the contrary that a circle $C$ contains a non-trivial self-affine set $E$. Let $f$ be a contractive affine map in the defining iterated function system of $E$. Then $f(E) \subset E$ and thus $f(E)$ is contained in both $C$ and $f(C)$. However, since $f(C)$ is an ellipse with diameter strictly smaller than that of $C$, the intersection of $f(C)$ and $C$ contains at most two points. This is a contradiction since $f(E)$ is an infinite set.

The above general phenomena was first clarified by Mattila [6] in the self-similar case. He proved that a self-similar set $E$ satisfying the open set condition either lies on an $m$-dimensional affine subspace or $\mathcal{H}^{t}(E \cap M)=0$ for every $m$-dimensional $C^{1}$-submanifold of $\mathbf{R}^{n}$. Here $t$ is the Hausdorff dimension of $E$ and $\mathcal{H}^{t}$ is the $t$ dimensional Hausdorff measure. This result was later generalized to self-conformal sets in $[4,5,7]$. As a related work, Bandt and Kravchenko [1] showed that if $E$ is a self-similar set which spans $\mathbf{R}^{n}$ and $x \in E$, then there does not exist a tangent hyperplane of $E$ at $x$.

As an easy consequence of the result of Mattila or that of Bandt and Kravchenko, an analytic planar curve does not contain any non-trivial self-similar set unless it is a straight line segment. In a private communication, Mattila asked which kind of analytic planar curves can contain a non-trivial self-affine set. The main purpose of this article is to answer this question.

We first remark that any closed parabolic arc is a self-affine set. This interesting fact was first pointed out by Bandt and Kravchenko [1]. In that paper, they considered self-affine planar curves consisting of two pieces $E=f_{1}(E) \cup f_{2}(E)$. They showed that if a certain condition on the eigenvalues of $f_{1}$ and $f_{2}$ holds, then the

https://doi.org/10.5186/aasfm.2018.4306

2010 Mathematics Subject Classification: Primary 28A80; Secondary 32C25, 51H30.

Key words: Self-affine set, analytic curve, algebraic surface. 
curve $E$ is differentiable at all except for countably many points. They also introduced a stronger condition on the eigenvalues which guarantees the curve $E$ to be continuously differentiable. This result implies that there exist many continuously differentiable self-affine curves. However, Bandt and Kravchenko furthermore showed that self-affine curves cannot be very smooth: the only simple $C^{2}$ self-affine planar curves are parabolic arcs and straight lines.

In our main result, instead of self-affine curves, we consider general self-affine sets and examine when they can be contained in an analytic curve.

Theorem A. An analytic curve in $\mathbf{R}^{n}, n \geq 2$, which cannot be embedded in a hyperplane contains a non-trivial self-affine set if and only if it is an affine image of $\eta:[c, d] \rightarrow \mathbf{R}^{n}, \eta(t)=\left(t, t^{2}, \ldots, t^{n}\right)$, for some $c<d$.

The above result gives a complete answer to the question of Mattila: the only analytic planar curves that contain non-trivial self-affine sets are parabolic arcs and straight line segments. As explained by Mattila, the question is related to the study of singular integrals and self-similar sets in Heisenberg groups. In such groups, selfsimilar sets are self-affine in the Euclidean metric. From the singular integral theory point of view, it is thus important to understand when a self-affine set is contained in an analytic manifold.

Concerning manifolds, we study an analogue of Mattila's question. We examine which kind of algebraic surfaces can contain self-affine sets. Our result shows that this cannot happen on compact surfaces.

Theorem B. A compact algebraic surface does not contain non-trivial self-affine sets.

It is easy to see that non-compact surfaces, such as paraboloids, can contain non-trivial self-affine sets; see Example 4.2. To finish the article, we introduce in Proposition 4.4 a sufficient condition for the inclusion of a self-affine set in an algebraic surface.

\section{Preliminaries}

In this section, we introduce the basic concepts to be used throughout in the article. A mapping $f: \mathbf{R}^{n} \rightarrow \mathbf{R}^{n}$ is affine if $f(x)=T x+c$ for all $x \in \mathbf{R}^{n}$, where $T$ is a $n \times n$ matrix and $c \in \mathbf{R}^{n}$. The matrix $T$ is called the linear part of $f$. It is easy to see that an affine map is invertible if and only if its linear part is non-singular. A mapping $f: \mathbf{R}^{n} \rightarrow \mathbf{R}^{n}$ is strictly contractive if $|f(x)-f(y)|<|x-y|$ for all $x, y \in \mathbf{R}^{n}$. Note that an affine mapping $f$ is strictly contractive if and only if its linear part $T$ has operator norm $\|T\|$ strictly less than 1 . A non-empty compact set $E \subset \mathbf{R}^{n}$ is called self-affine if $E=\bigcup_{i=1}^{\ell} f_{i}(E)$, where $\left\{f_{i}\right\}_{i=1}^{\ell}$ is an affine iterated function system (IFS), i.e. a finite collection of strictly contractive invertible affine maps $f_{i}: \mathbf{R}^{n} \rightarrow \mathbf{R}^{n}$; see [3]. Moreover, $E$ is called self-similar if all the $f_{i}$ 's are similitudes. We say that a self-affine set is non-trivial if it is not a singleton.

If $a<b$, then a non-constant continuous function $\gamma:[a, b] \rightarrow \mathbf{R}^{n}$ is called a curve. We denote the set $\gamma([a, b]) \subset \mathbf{R}^{n}$ by $\operatorname{Img}(\gamma)$ and refer to it also as a curve. By saying that a curve $\gamma$ contains a set $A$ we obviously mean that $A \subset \operatorname{Img}(\gamma)$. A curve $\gamma$ is simple if $\gamma(s) \neq \gamma(t)$ for $a \leq s<t<b$. We say that a curve $\gamma:[a, b] \rightarrow \mathbf{R}^{n}$, $\gamma(t)=\left(x_{1}(t), \ldots, x_{n}(t)\right)$, is analytic if $x_{i}:[a, b] \rightarrow \mathbf{R}$ is continuous on $[a, b]$ and real analytic on $(a, b)$ for all $i \in\{1, \ldots, n\}$. Recall that a function is real analytic on an open set $U \subset \mathbf{R}$ if, at any point $t \in U$, it can be represented by a convergent 
power series on some interval of positive radius centered at $t$. Similarly, if $x_{i}$ 's are $C^{k}$ functions for some $k \in \mathbf{N}$, then the curve $\gamma$ is called $C^{k}$ curve. The $k$-th derivative of a $C^{k}$ curve $\gamma$ is $\gamma^{(k)}(t)=\left(x_{1}^{(k)}(t), \ldots, x_{n}^{(k)}(t)\right)$. If $f: \mathbf{R}^{n} \rightarrow \mathbf{R}^{n}$ is an invertible affine mapping and $\gamma:[a, b] \rightarrow \mathbf{R}^{n}$ is a curve, then $f \circ \gamma$ is the affine image of the curve.

Let $P: \mathbf{R}^{n} \rightarrow \mathbf{R}$ be a non-constant polynomial with real coefficients. The set

$$
S(P)=\left\{x \in \mathbf{R}^{n}: P(x)=0\right\}
$$

is called an algebraic surface. The degree of $P$, denoted by $\operatorname{deg}(P)$, is the highest degree of its terms, when $P$ is expressed in canonical form. The degree of a term is the sum of the exponents of the variables that appear in it.

\section{Self-affine sets and analytic curves}

In this section, we prove Theorem A. Our arguments are inspired by the proof of [1, Theorem 3(i)]. We will first show that an affine image of $\eta:[c, d] \rightarrow \mathbf{R}^{n}$, $\eta(t)=\left(t, t^{2}, \ldots, t^{n}\right)$, contains a non-trivial self-affine set. This follows immediately from the following lemma.

Lemma 3.1. If $\eta:[c, d] \rightarrow \mathbf{R}^{n}$ is given by $\eta(t)=\left(t, t^{2}, \ldots, t^{n}\right)$, then $\operatorname{Img}(\eta)$ is a non-trivial self-affine set for all $c<d$.

Proof. Let

$$
0<\lambda<\left(2^{n} \sqrt{n} \max \left\{(2|c|+1)^{n},(|c|+|d|+1)^{n}\right\}\right)^{-1}<1
$$

and choose $t_{1}, \ldots, t_{\ell} \in[c, d]$ with $\ell \in \mathbf{N}$ such that the self-similar set of $\{x \mapsto$ $\left.\lambda(x-c)+t_{i}\right\}_{i=1}^{\ell}$ is $[c, d]$. Write $c_{i, k, j}=\left(\begin{array}{l}k \\ j\end{array}\right)\left(\frac{t_{i}}{\lambda}-c\right)^{k-j}$ and observe that

$$
\left(t-\left(c-\frac{t_{i}}{\lambda}\right)\right)^{k}=\sum_{j=1}^{k} c_{i, k, j}\left(t^{j}-\left(c-\frac{t_{i}}{\lambda}\right)^{j}\right)
$$

for all $k \in\{1, \ldots, n\}, i \in\{1, \ldots, \ell\}$, and $t \in \mathbf{R}$.

Defining for each $i \in\{1, \ldots, \ell\}$ a lower-triangular matrix by

$$
T_{i}=\left(\begin{array}{ccccc}
\lambda c_{i, 1,1} & 0 & 0 & \cdots & 0 \\
\lambda^{2} c_{i, 2,1} & \lambda^{2} c_{i, 2,2} & 0 & \cdots & 0 \\
\lambda^{3} c_{i, 3,1} & \lambda^{3} c_{i, 3,2} & \lambda^{3} c_{i, 3,3} & \cdots & 0 \\
\vdots & \vdots & \vdots & \ddots & \vdots \\
\lambda^{n} c_{i, n, 1} & \lambda^{n} c_{i, n, 2} & \lambda^{n} c_{i, n, 3} & \cdots & \lambda^{n} c_{i, n, n}
\end{array}\right),
$$

we see, by the choice of $\lambda$ and the fact that $t_{i} \in[c, d]$, that

$$
\begin{aligned}
\left\|T_{i}\right\| & \leq \sqrt{n} \max _{k \in\{1, \ldots, n\}} \sum_{j=1}^{k}\left|\lambda^{k} c_{i, k, j}\right|=\sqrt{n} \max _{k \in\{1, \ldots, n\}} \sum_{j=1}^{k} \lambda^{k}\left(\begin{array}{c}
k \\
j
\end{array}\right)\left|\frac{t_{i}}{\lambda}-c\right|^{k-j} \\
& \leq \sqrt{n} \max _{k \in\{1, \ldots, n\}} \sum_{j=1}^{k} \lambda^{j}\left(\begin{array}{c}
k \\
j
\end{array}\right)\left(\left|t_{i}\right|+|c|+1\right)^{k-j} \leq \lambda \sqrt{n} \max _{k \in\{1, \ldots, n\}}\left(\left|t_{i}\right|+|c|+1\right)^{k} 2^{k}<1 .
\end{aligned}
$$

Therefore, the affine map $f_{i}: \mathbf{R}^{n} \rightarrow \mathbf{R}^{n}$ defined by

$$
f_{i}\left(x_{1}, \ldots, x_{n}\right)=T_{i}\left(x_{1}, \ldots, x_{n}\right)-T_{i}\left(c-\frac{t_{i}}{\lambda},\left(c-\frac{t_{i}}{\lambda}\right)^{2}, \ldots,\left(c-\frac{t_{i}}{\lambda}\right)^{n}\right)
$$


is contractive and satisfies

$$
\begin{aligned}
f_{i}\left(t, t^{2}, \ldots, t^{n}\right) & =T_{i}\left(t-\left(c-\frac{t_{i}}{\lambda}\right), t^{2}-\left(c-\frac{t_{i}}{\lambda}\right)^{2}, \ldots, t^{n}-\left(c-\frac{t_{i}}{\lambda}\right)^{n}\right) \\
& =\left(\lambda\left(t-\left(c-\frac{t_{i}}{\lambda}\right)\right), \lambda^{2}\left(t-\left(c-\frac{t_{i}}{\lambda}\right)\right)^{2}, \ldots, \lambda^{n}\left(t-\left(c-\frac{t_{i}}{\lambda}\right)\right)^{n}\right) \\
& =\left(\lambda(t-c)+t_{i},\left(\lambda(t-c)+t_{i}\right)^{2}, \ldots,\left(\lambda(t-c)+t_{i}\right)^{n}\right)
\end{aligned}
$$

for all $t \in[c, d]$. Hence the self-affine set of $\left\{f_{i}\right\}_{i=1}^{\ell}$ is the curve $\operatorname{Img}(\eta)$.

Remark 3.2. The key fact implicitly used in the above proof is that $\eta(t)=$ $\left(t, t^{2}, \ldots, t^{n}\right)$ defined on $\mathbf{R}$ is invariant under homotheties $\operatorname{diag}\left(s, s^{2}, \ldots, s^{n}\right)$ and translations $\left(t, t^{2}, \ldots, t^{n}\right) \mapsto\left(t-a,(t-a)^{2}, \ldots,(t-a)^{n}\right)$.

Let us next focus on the opposite claim.

Theorem 3.3. If an analytic curve which cannot be embedded in a hyperplane contains a non-trivial self-affine set, then it is an affine image of $\eta:[c, d] \rightarrow \mathbf{R}^{n}$, $\eta(t)=\left(t, t^{2}, \ldots, t^{n}\right)$, for some $c<d$.

Proof. Let $\gamma:[a, b] \rightarrow \mathbf{R}^{n}$ be an analytic curve such that $\operatorname{Img}(\gamma)$ is not contained in a hyperplane. Suppose that $E$ is a non-trivial self-affine set of an affine IFS $\left\{f_{i}\right\}_{i=1}^{\ell}$ such that $E \subset \operatorname{Img}(\gamma)$. Let $\mathcal{S}$ be the semigroup generated by $f_{1}, \ldots, f_{\ell}$ under composition.

By analyticity and the assumption that $\operatorname{Img}(\gamma)$ is not contained in a hyperplane, without loss of generality, we may assume that $E \subset \gamma((a, b))$ and $\gamma^{\prime}(t) \neq 0$ for all $t \in(a, b)$. Since $(a, b)$ has a countable cover of open intervals $I_{i}$ such that $\gamma\left(I_{i}\right)$ has no intersection points, we have $E \subset \bigcup_{i} E \cap \gamma\left(I_{i}\right)$ and therefore, by the Baire Category Theorem, there exist $i$ and an open set $U$ such that $\emptyset \neq E \cap U \subset E \cap \gamma\left(I_{i}\right)$. Since $E \cap U$ contains a non-trivial self-affine set, we see that no generality is lost if we assume the curve $\gamma$ to be simple.

Fix $\varphi \in \mathcal{S}$ and write

$$
\varphi(x)=M\left(x-x_{0}\right)+x_{0}
$$

for all $x \in \mathbf{R}^{n}$, where $x_{0} \in \mathbf{R}^{n}$ is the fixed point of $\varphi$ and $M$ is an $n \times n$ invertible matrix. Note that $x_{0} \in E$. Since $E \subset \gamma((a, b))$ there exists $t_{0} \in(a, b)$ such that $x_{0}=\gamma\left(t_{0}\right)$. Hence we may rewrite $(3.1)$ as

$$
\varphi(x)=M\left(x-\gamma\left(t_{0}\right)\right)+\gamma\left(t_{0}\right) .
$$

Since $E$ is non-trivial, there exists a sequence $\left(t_{i}\right)_{i \in \mathbf{N}}$ of distinct numbers in $(a, b)$ such that $t_{i} \rightarrow t_{0}$ as $i \rightarrow \infty$ and $\gamma\left(t_{i}\right) \in E$ for all $i \in \mathbf{N}$. Furthermore, since $\varphi(E) \subset E \subset \gamma((a, b))$, we see that $\varphi\left(\gamma\left(t_{i}\right)\right) \in \operatorname{Img}(\gamma)$ and therefore, for each $i \in \mathbf{N}$ there exists $t_{i}^{\prime} \in(a, b)$ such that

$$
\varphi\left(\gamma\left(t_{i}\right)\right)=\gamma\left(t_{i}^{\prime}\right)
$$

Recalling that $\gamma$ is simple and $\varphi\left(\gamma\left(t_{0}\right)\right)=\gamma\left(t_{0}\right)$, we see that $t_{i}^{\prime} \rightarrow t_{0}$ as $i \rightarrow \infty$. By (3.2) and (3.3), we have

$$
M\left(\gamma\left(t_{i}\right)-\gamma\left(t_{0}\right)\right)=\varphi\left(\gamma\left(t_{i}\right)\right)-\gamma\left(t_{0}\right)=\gamma\left(t_{i}^{\prime}\right)-\gamma\left(t_{0}\right)
$$

and therefore,

$$
M\left(\frac{\gamma\left(t_{i}\right)-\gamma\left(t_{0}\right)}{t_{i}-t_{0}}\right)=\frac{\gamma\left(t_{i}^{\prime}\right)-\gamma\left(t_{0}\right)}{t_{i}^{\prime}-t_{0}} \cdot \frac{t_{i}^{\prime}-t_{0}}{t_{i}-t_{0}}
$$


Letting $i \rightarrow \infty$, we have

$$
M \gamma^{\prime}\left(t_{0}\right)=\lambda \gamma^{\prime}\left(t_{0}\right),
$$

where $\lambda=\lim _{i \rightarrow \infty}\left(t_{i}^{\prime}-t_{0}\right) /\left(t_{i}-t_{0}\right) \neq 0$ by the invertibility of $M$.

Let $J$ be an invertible matrix such that

$$
J^{-1} \gamma^{\prime}\left(t_{0}\right)=(1,0, \ldots, 0)
$$

and

$$
J^{-1} M J=\left(\begin{array}{cccc}
A_{1} & 0 & \cdots & 0 \\
0 & A_{2} & \cdots & 0 \\
\vdots & \vdots & \ddots & \vdots \\
0 & 0 & \cdots & A_{m}
\end{array}\right)
$$

is a real canonical Jordan form of $M$. Write $A=J^{-1} M J$ and recall that if $\lambda_{i}$ is a real eigenvalue of $M$, then

$$
A_{i}=\left(\begin{array}{cccccc}
\lambda_{i} & 1 & 0 & \cdots & 0 & 0 \\
0 & \lambda_{i} & 1 & \cdots & 0 & 0 \\
0 & 0 & \lambda_{i} & \cdots & 0 & 0 \\
\vdots & \vdots & \vdots & \ddots & \vdots & \vdots \\
0 & 0 & 0 & \cdots & \lambda_{i} & 1 \\
0 & 0 & 0 & \cdots & 0 & \lambda_{i}
\end{array}\right)
$$

and if $\lambda_{i}$ is a non-real eigenvalue of $M$ with real part $a_{i}$ and imaginary part $b_{i}$, then

$$
A_{i}=\left(\begin{array}{cccccc}
C_{i} & I & 0 & \cdots & 0 & 0 \\
0 & C_{i} & I & \cdots & 0 & 0 \\
0 & 0 & C_{i} & \cdots & 0 & 0 \\
\vdots & \vdots & \vdots & \ddots & \vdots & \vdots \\
0 & 0 & 0 & \cdots & C_{i} & I \\
0 & 0 & 0 & \cdots & 0 & C_{i}
\end{array}\right)
$$

where

$$
C_{i}=\left(\begin{array}{cc}
a_{i} & b_{i} \\
-b_{i} & a_{i}
\end{array}\right) \text { and } I=\left(\begin{array}{cc}
1 & 0 \\
0 & 1
\end{array}\right) .
$$

Note that by (3.5), we have $\lambda_{1}=\lambda \in \mathbf{R}$. Moreover by (3.4),

$$
A J^{-1}\left(\gamma\left(t_{i}\right)-\gamma\left(t_{0}\right)\right)=J^{-1}\left(\gamma\left(t_{i}^{\prime}\right)-\gamma\left(t_{0}\right)\right)
$$

for all $i \in \mathbf{N}$.

Defining $\tilde{\gamma}:[a, b] \rightarrow \mathbf{R}^{n}$ by

$$
\tilde{\gamma}(t)=J^{-1}\left(\gamma(t)-\gamma\left(t_{0}\right)\right)
$$

we clearly have $\tilde{\gamma}\left(t_{0}\right)=0$ and $\tilde{\gamma}^{\prime}\left(t_{0}\right)=J^{-1} \gamma^{\prime}\left(t_{0}\right)=(1,0, \ldots, 0)$. Write $\tilde{\gamma}(t)=$ $\left(\tilde{x}_{1}(t), \ldots, \tilde{x}_{n}(t)\right)$. Then $\tilde{x}_{1}\left(t_{0}\right)=0$ and $\tilde{x}_{1}^{\prime}\left(t_{0}\right)=1 \neq 0$. By the inverse function theorem, the function $\tilde{x}_{1}(t)$ has a local inverse $t=t\left(\tilde{x}_{1}\right)$ which is analytic on $(-\varepsilon, \varepsilon)$ for some $\varepsilon>0$. Write $x_{1}^{*}=\tilde{x}_{1}$ and $x_{k}^{*}\left(x_{1}^{*}\right)=\tilde{x}_{k}\left(t\left(x_{1}^{*}\right)\right)$ for $k \in\{2, \ldots, n\}$. Clearly $x_{k}^{*}(\cdot)$ is analytic on $(-\varepsilon, \varepsilon)$ for all $k \in\{2, \ldots, n\}$. Note that

$$
x_{k}^{*}(0)=\tilde{x}_{k}\left(t_{0}\right)=0, \quad\left(x_{k}^{*}\right)^{\prime}(0)=\tilde{x}_{k}^{\prime}\left(t_{0}\right) \cdot t^{\prime}(0)=0
$$

for all $k \in\{2, \ldots, n\}$ and $x_{2}^{*}, \ldots, x_{n}^{*}$ are not constant functions. Indeed, if $x_{k}^{*}$ is constant for some $k$, then so is $\tilde{x}_{k}$; by the fact that each $\tilde{x}_{k}$ is a linear combination of 
$x_{1}, \ldots, x_{n}$ (see (3.7)), the curve $\gamma$ would be contained in a hyperplane in $\mathbf{R}^{n}$, leading to a contradiction. Let $\xi:(-\varepsilon, \varepsilon) \rightarrow \mathbf{R}^{n}$ be defined by

$$
\xi\left(x_{1}^{*}\right)=\left(x_{1}^{*}, x_{2}^{*}\left(x_{1}^{*}\right), \ldots, x_{n}^{*}\left(x_{1}^{*}\right)\right) .
$$

Then $\xi$ is a re-parametrization of the curve $\tilde{\gamma}$ restricted on a neighborhood of $t_{0}$. The goal of the proof is to show that an affine image of the curve $\xi$ will be of the claimed form.

Let us next collect three facts related to the above defined setting.

Fact 1 . Write $A=\left(a_{i j}\right)_{1 \leq i, j \leq n}$ and let $Y=a_{11} x_{1}^{*}+\sum_{j=2}^{n} a_{1 j} x_{j}^{*}\left(x_{1}^{*}\right)$. Then

$$
A\left(x_{1}^{*}, x_{2}^{*}\left(x_{1}^{*}\right), \ldots, x_{n}^{*}\left(x_{1}^{*}\right)\right)=\left(Y, x_{2}^{*}(Y), \ldots, x_{n}^{*}(Y)\right)
$$

for all $x_{1}^{*} \in(-\varepsilon, \varepsilon)$.

Proof. By (3.6), $A \tilde{\gamma}\left(t_{i}\right)=\tilde{\gamma}\left(t_{i}^{\prime}\right)$ for all $i \in \mathbf{N}$. Hence the equality (3.10) holds for infinitely many different values of $x_{1}^{*}$ in a small closed neighborhood of 0 . By analyticity, (3.10) holds on the whole interval $(-\varepsilon, \varepsilon)$.

The next fact concerns the shape of the matrix $A$.

Fact 2. The matrix $A$ is diagonal. In other words, all the block matrices $A_{i}$ have dimension 1.

Proof. Let us first show that $A_{1}$ has dimension 1. Suppose to the contrary that $d_{1}=\operatorname{dim}\left(A_{1}\right)>1$. Since the eigenvalue associated to $A_{1}$ is $\lambda \in \mathbf{R}$, we have

$$
A_{1}=\left(\begin{array}{ccccc}
\lambda & 1 & \cdots & 0 & 0 \\
0 & \lambda & \cdots & 0 & 0 \\
\vdots & \vdots & \ddots & \vdots & \vdots \\
0 & 0 & \cdots & \lambda & 1 \\
0 & 0 & \cdots & 0 & \lambda
\end{array}\right)
$$

By Fact 1, we see that

$$
\lambda x_{d_{1}}^{*}\left(x_{1}^{*}\right)=x_{d_{1}}^{*}\left(\lambda x_{1}^{*}+x_{2}^{*}\left(x_{1}^{*}\right)\right) .
$$

By (3.8) and the fact that $x_{k}^{*}, k \in\{2, \ldots, n\}$, is not a constant, there exist integers $p_{2}, \ldots, p_{n} \geq 2$ and reals $c_{2}, \ldots, c_{n} \neq 0$ such that for each $k \in\{2, \ldots, n\}$

$$
x_{k}^{*}\left(x_{1}^{*}\right)=c_{k}\left(x_{1}^{*}\right)^{p_{k}}+o\left(x_{1}^{*}\right)^{p_{k}}
$$

as $x_{1}^{*} \rightarrow 0$. Plugging (3.12) into (3.11), and comparing the coefficients of Taylor series in $x_{1}^{*}$ on both sides, we get

$$
\lambda c_{d_{1}}=c_{d_{1}} \lambda^{p_{d_{1}}}
$$

which implies that $p_{d_{1}}=1$, a contradiction. Hence we have $\operatorname{dim}\left(A_{1}\right)=1$ and therefore $Y=\lambda x_{1}^{*}$.

Let us next assume inductively that for some $k \in\{1, \ldots, n-1\}$ the matrices $A_{1}, \ldots, A_{k}$ are of dimension 1 and show that $\operatorname{dim}\left(A_{k+1}\right)=1$. Suppose to the contrary that $d=\operatorname{dim}\left(A_{k+1}\right)>1$. Now there are two cases: either $\lambda_{k+1}$ is real or not. First suppose that $\lambda_{k+1}$ is real. Let $\ell=k+d$. By (3.10) we have

$$
\begin{aligned}
\lambda_{k+1} x_{\ell-1}^{*}\left(x_{1}^{*}\right)+x_{\ell}^{*}\left(x_{1}^{*}\right) & =x_{\ell-1}^{*}\left(\lambda x_{1}^{*}\right), \\
\lambda_{k+1} x_{\ell}^{*}\left(x_{1}^{*}\right) & =x_{\ell}^{*}\left(\lambda x_{1}^{*}\right) .
\end{aligned}
$$


Plugging (3.12) into (3.14), and comparing the coefficients of Taylor series in $x_{1}^{*}$ on both sides, we get $\lambda_{k+1}=\lambda^{p_{\ell}}$. Then plug (3.12) into (3.13) to obtain

$$
\lambda^{p_{\ell}} c_{\ell-1}\left(x_{1}^{*}\right)^{p_{\ell-1}}+c_{\ell}\left(x_{1}^{*}\right)^{p_{\ell}}=\lambda^{p_{\ell-1}} c_{\ell-1}\left(x_{1}^{*}\right)^{p_{\ell-1}}+o\left(\left(x_{1}^{*}\right)^{p_{\ell-1}}+\left(x_{1}^{*}\right)^{p_{\ell}}\right)
$$

as $x_{1}^{*} \rightarrow 0$. That is,

$$
\left(\lambda^{p_{\ell}}-\lambda^{p_{\ell-1}}\right) c_{\ell-1}\left(x_{1}^{*}\right)^{p_{\ell-1}}+c_{\ell}\left(x_{1}^{*}\right)^{p_{\ell}}=o\left(\left(x_{1}^{*}\right)^{p_{\ell-1}}+\left(x_{1}^{*}\right)^{p_{\ell}}\right)
$$

as $x_{1}^{*} \rightarrow 0$. Since $0<|\lambda|<1$ and $c_{\ell-1}, c_{\ell} \neq 0$, one easily derives a contradiction from (3.15) by considering the cases $p_{\ell}<p_{\ell-1}, p_{\ell}=p_{\ell-1}$, and $p_{\ell}>p_{\ell-1}$ separately.

Hence we may assume that $\lambda_{k+1}=a+i b$ with $b \neq 0$. The matrix $A_{k+1}$ is therefore of the form

$$
A_{k+1}=\left(\begin{array}{ccccccc}
a & b & 1 & 0 & \cdots & 0 & 0 \\
-b & a & 0 & 1 & \cdots & 0 & 0 \\
0 & 0 & a & b & \cdots & 0 & 0 \\
0 & 0 & -b & a & \cdots & 0 & 0 \\
\vdots & \vdots & \vdots & \vdots & \ddots & \vdots & \vdots \\
0 & 0 & 0 & 0 & \cdots & a & b \\
0 & 0 & 0 & 0 & \cdots & -b & a
\end{array}\right) .
$$

Again let $\ell=k+d$. Applying (3.10), we see that

$$
\begin{aligned}
a x_{\ell-1}^{*}\left(x_{1}^{*}\right)+b x_{\ell}^{*}\left(x_{1}^{*}\right) & =x_{\ell-1}^{*}\left(\lambda x_{1}^{*}\right), \\
-b x_{\ell-1}^{*}\left(x_{1}^{*}\right)+a x_{\ell}^{*}\left(x_{1}^{*}\right) & =x_{\ell}^{*}\left(\lambda x_{1}^{*}\right) .
\end{aligned}
$$

Using the above identities and comparing the coefficients of $\left(x_{1}^{*}\right)^{p_{\ell}}$ and $\left(x_{1}^{*}\right)^{p_{\ell-1}}$ in the Taylor expansions of $x_{\ell}^{*}$ and $x_{\ell-1}^{*}$, we see that $p_{\ell}=p_{\ell-1}$; and moreover,

$$
\begin{aligned}
a c_{\ell-1}+b c_{\ell} & =c_{\ell-1} \lambda^{p_{\ell}}, \\
-b c_{\ell-1}+a c_{\ell} & =c_{\ell} \lambda^{p_{\ell}},
\end{aligned}
$$

or, equivalently,

$$
\left(\begin{array}{cc}
a & b \\
-b & a
\end{array}\right)\left(\begin{array}{c}
c_{\ell-1} \\
c_{\ell}
\end{array}\right)=\lambda^{p_{\ell}}\left(\begin{array}{c}
c_{\ell-1} \\
c_{\ell}
\end{array}\right)
$$

This means that the real number $\lambda^{p_{\ell}}$ is an eigenvalue of the above matrix, a contradiction.

By Fact 2, we may now write

$$
A=\operatorname{diag}\left(\lambda_{1}, \lambda_{2}, \ldots, \lambda_{n}\right),
$$

where $\lambda_{1}=\lambda \in(-1,1) \backslash\{0\}$. With this observation, we can examine how the curve $\xi$ defined in (3.9) looks like.

Fact 3. There exist integers $2 \leq p_{2}<p_{3}<\cdots<p_{n}$ such that a piece of the curve $\operatorname{Img}(\gamma)$, namely $\gamma:\left(t_{0}-\delta_{1}, t_{0}+\delta_{2}\right) \rightarrow \mathbf{R}^{n}$ for some $\delta_{1}, \delta_{2}>0$, is an affine image of the curve $\eta:(-\varepsilon, \varepsilon) \rightarrow \mathbf{R}^{n}$ defined by

$$
\eta(t)=\left(t, t^{p_{2}}, \ldots, t^{p_{n}}\right) .
$$

More precisely, there exists an invertible $n \times n$ matrix $B$ such that the above defined $\eta$ is the re-parametrization of the curve $B\left(\gamma(t)-\gamma\left(t_{0}\right)\right), t \in\left(t_{0}-\delta_{1}, t_{0}+\delta_{2}\right)$.

Proof. We first examine the curve $\xi$ defined in (3.9). By (3.16) and (3.10), we have for $2 \leq k \leq n$,

$$
x_{k}^{*}\left(\lambda x_{1}^{*}\right)=\lambda_{k} x_{k}^{*}\left(x_{1}^{*}\right)
$$


and hence, by (3.12), there exist integers $p_{2}, \ldots, p_{n} \geq 2$ and reals $c_{2}, \ldots, c_{n} \neq 0$ such that

$$
c_{k}\left(\lambda x_{1}^{*}\right)^{p_{k}}=\lambda_{k} c_{k}\left(x_{1}^{*}\right)^{p_{k}}+o\left(\left(x_{1}^{*}\right)^{p_{k}}\right) .
$$

This implies that $\lambda_{k}=\lambda^{p_{k}}$ and thus $x_{k}^{*}\left(\lambda x_{1}^{*}\right)=\lambda^{p_{k}} x_{k}^{*}\left(x_{1}^{*}\right)$. Taking $p_{k}$-th derivative on both sides gives $\left(x_{k}^{*}\right)^{\left(p_{k}\right)}\left(\lambda x_{1}^{*}\right)=\left(x_{k}^{*}\right)^{\left(p_{k}\right)}\left(x_{1}^{*}\right)$. Hence $\left(x_{k}^{*}\right)^{\left(p_{k}\right)}\left(\lambda^{j} x_{1}^{*}\right)=\left(x_{k}^{*}\right)^{\left(p_{k}\right)}\left(x_{1}^{*}\right)$ for all $j \in \mathbf{N}$. Letting $j \rightarrow \infty$, we get $\left(x_{k}^{*}\right)^{\left(p_{k}\right)}\left(x_{1}^{*}\right) \equiv\left(x_{k}^{*}\right)^{\left(p_{k}\right)}(0)=c_{k} p_{k}$ !. Combining this with (3.12) yields

$$
x_{k}^{*}\left(x_{1}^{*}\right)=c_{k}\left(x_{1}^{*}\right)^{p_{k}} .
$$

Since the curve $\tilde{\gamma}$ is not contained in a hyperplane, we see that, for any nonzero vector $\left(b_{1}, \ldots, b_{n}\right)$, the sum $\sum_{k=1}^{n} b_{k} x_{k}^{*}$ is not identically zero. Thus the integers $p_{2}, \ldots, p_{n}$ are mutually distinct. Hence the curve $\xi:(-\epsilon, \epsilon) \rightarrow \mathbf{R}^{n}$ is of the form $\xi\left(x_{1}^{*}\right)=\left(x_{1}^{*}, c_{2}\left(x_{1}^{*}\right)^{p_{2}}, \ldots, c_{n}\left(x_{1}^{*}\right)^{p_{n}}\right)$. Without confusion, we simply write $\xi(t)=\left(t, c_{2} t^{p_{2}}, \ldots, c_{n} t^{p_{n}}\right)$.

We have now proved that, possibly after a permutation on coordinate axis, the curve $\tilde{\gamma}:\left(t_{0}-\delta_{1}, t_{0}+\delta_{2}\right) \rightarrow \mathbf{R}^{n}$ for some $\delta_{1}, \delta_{2}>0$, can be re-pararemtrized by

$$
t \mapsto\left(t, c_{2} t^{p_{2}}, \ldots, c_{n} t^{p_{n}}\right), t \in(-\epsilon, \epsilon)
$$

for some integers $2 \leq p_{2}<p_{3}<\cdots<p_{n}$ and reals $c_{2}, \ldots, c_{n} \neq 0$. Applying a further affine transformation $\left(u_{1}, u_{2}, \ldots, u_{n}\right) \mapsto\left(u_{1}, u_{2} / c_{2}, \ldots, u_{n} / c_{n}\right)$, we see that $\gamma:\left(t_{0}-\delta_{1}, t_{0}+\delta_{2}\right) \rightarrow \mathbf{R}^{n}$, for some $\delta_{1}, \delta_{2}>0$, is an affine image of the curve $\eta$. This completes the proof of Fact 3 .

By Fact 3, it suffices to show that $p_{k}=k$ for all $k \in\{2, \ldots, n\}$. Observe that $\eta:(-\varepsilon, \varepsilon) \rightarrow \mathbf{R}^{n}$ given by Fact 3 is an analytic simple curve which cannot be embedded in a hyperplane and it contains a non-trivial self-affine set, say $F$. Then there exists $t_{1} \in(-\varepsilon, \varepsilon) \backslash\{0\}$ such that $\eta\left(t_{1}\right)$ is the fixed point of a mapping of the affine IFS defining $F$. Therefore, applying the previous argument (Fact 3 ) once more (in which $\gamma$ is replaced by $\eta$ ), we find integers $2 \leq q_{2}<q_{3}<\cdots<q_{n}$ and an interval $\left(t_{1}-\delta_{1}^{\prime}, t_{1}+\delta_{2}^{\prime}\right) \subset(-\epsilon, \epsilon)$ for some some $\delta_{1}^{\prime}, \delta_{2}^{\prime}>0$ such that, under a suitable invertible linear transformation $B^{\prime}$, the curve

$$
t \mapsto B^{\prime}\left(\eta(t)-\eta\left(t_{1}\right)\right)
$$

defined on $\left(t_{1}-\delta_{1}^{\prime}, t_{1}+\delta_{2}^{\prime}\right)$ can be re-parametrized by

$$
t \mapsto\left(t, t^{q_{2}}, \ldots, t^{q_{n}}\right) .
$$

This means that, writing $B^{\prime}=\left(b_{k j}\right)_{1 \leq k, j \leq n}$, we have

$$
\sum_{j=1}^{n} b_{k j}\left(t^{p_{j}}-t_{1}^{p_{j}}\right)=\left(\sum_{j=1}^{n} b_{1 j}\left(t^{p_{j}}-t_{1}^{p_{j}}\right)\right)^{q_{k}}
$$

for all $t \in\left(t_{1}-\delta_{1}^{\prime}, t_{1}+\delta_{2}^{\prime}\right)$ and $k \in\{2, \ldots, n\}$, where $p_{1}=1$. By analyticity, (3.18) holds for all $t \in \mathbf{R}$.

We will next compare the degrees of polynomials of $t$ on both sides of (3.18) for all $k \in\{2, \ldots, n\}$. Let $d=\operatorname{deg}\left(\sum_{j=1}^{n} b_{1 j}\left(t^{p_{j}}-t_{1}^{p_{j}}\right)\right) \in\left\{1, p_{2}, \ldots, p_{n}\right\}$. When $k$ runs over $\{2, \ldots, n\}$, the degrees of the right-hand side of $(3.18)$ are $d q_{2}, d q_{3}, \ldots, d q_{n}$, whereas the left-hand side has degree in $\left\{1, p_{2}, \ldots, p_{n}\right\}$. Therefore,

$$
\left\{d q_{2}, d q_{3}, \ldots, d q_{n}\right\} \subset\left\{1, p_{2}, \ldots, p_{n}\right\}
$$

which implies that

$$
p_{k}=d q_{k}
$$


for all $k \in\{2, \ldots, n\}$. Since $d \in\left\{1, p_{2}, \ldots, p_{n}\right\}$, we must have $d=1$ (otherwise, by (3.19), $q_{k}=1$ for some $k \in\{2, \ldots, n\}$ which is a contradiction). But since $d=1$, we may write (3.18) as

$$
\sum_{j=1}^{n} b_{k j}\left(t^{p_{j}}-t_{1}^{p_{j}}\right)=\left(c\left(t-t_{1}\right)\right)^{p_{k}}
$$

for all $k \in\{2, \ldots, n\}$. In particular, this shows that $\left(t-t_{1}\right)^{p_{n}}$ is a linear combination of $\left(t-t_{1}\right),\left(t^{p_{2}}-t_{1}^{p_{2}}\right), \ldots,\left(t^{p_{n}}-t_{1}^{p_{n}}\right)$. Since $t_{1} \neq 0$, all powers $t^{j}, j \in\left\{1, \ldots, p_{n}\right\}$, appear in $\left(t-t_{1}\right)^{p_{n}}$ with non-degenerate coefficients, and it follows that $p_{k}=k$ for all $k \in\{2, \ldots, n\}$.

Remark 3.4. (1) Bandt and Kravchenko showed that there are plenty of $C^{1}$ planar self-affine curves (i.e. self-affine sets that are $C^{1}$ planar curves); see [1, Theorem 2]. Furthermore, in [1, Theorem 3(ii)], they showed that parabolic arcs and straight line segments are the only simple $C^{2}$ planar self-affine curves. This result also follows from Theorem A by a simple modification. It would be interesting to know that if a self-affine set $E$ is contained in a $C^{2}$ planar curve, then does there exists an analytic curve containing $E$ ?

(2) The analyticity assumption in Theorem A is well motivated since for each $k \in \mathbf{N}$ it is easy to construct a non-parabolic $C^{k}$ planar curve containing a self-affine set. To see this, start from a piece of parabolic curve and change a small part of it so that the new curve is $C^{k}$. Clearly the obtained curve still contains a self-affine set. Due to this, it would be interesting to know if there exists a self-affine set $E$ which is a subset of a strictly convex $C^{2}$ planar curve, but is not a subset of any parabolic curve. Also, when can a self-affine set intersect an analytic curve in a set of positive measure for some relevant measure such as the self-affine measure? In the self-conformal case, this property implies that the whole set is contained in an analytic curve; see [4, Theorem 2.1].

\section{Self-affine sets and algebraic surfaces}

In this section, we prove Theorem B and introduce self-affine polynomials.

Proof of Theorem $B$. Let $P: \mathbf{R}^{n} \rightarrow \mathbf{R}$ be a non-constant polynomial with real coefficients such that $S(P)$ is compact. Suppose to the contrary that there exists a non-trivial self-affine set $E$ contained in $S(P)$. Let $f$ be one of the mappings of the affine IFS defining $E$ and set $P_{j}=P \circ f^{-j}$ for all $j \in \mathbf{N}$. Observe that the degree of $P_{j}$ is at most $\operatorname{deg}(P)$. It is easy to see that $S\left(P_{j}\right)=f^{j}(S(P))$ for all $j \in \mathbf{N}$ and therefore $\operatorname{diam}\left(S\left(P_{j}\right)\right) \rightarrow 0$ as $j \rightarrow \infty$. By the assumption, we have $f^{j}(E) \subset f^{j}(S(P))=S\left(P_{j}\right)$ for all $j \in \mathbf{N}$, and by the invariance, we have $f^{j}(E) \subset f^{j-1}(E) \subset \cdots \subset E$ for all $j \in \mathbf{N}$.

Since the ring of polynomials having degree at most $\operatorname{deg}(P)$ is finite dimensional there exist $P_{k_{1}}, \ldots, P_{k_{m}}$ such that each $P_{j}$ is a linear combination of these polynomials. Choose $j$ so large that

$$
\operatorname{diam}\left(S\left(P_{j}\right)\right)<\min _{i \in\{1, \ldots, m\}} \operatorname{diam}\left(f^{k_{i}}(E)\right)=\operatorname{diam}\left(\bigcap_{i=1}^{m} f^{k_{i}}(E)\right) .
$$

But since $P_{j}=\sum_{i=1}^{m} c_{i} P_{k_{i}}$ for some $c_{i}$, we have

$$
\bigcap_{i=1}^{m} f^{k_{i}}(E) \subset \bigcap_{i=1}^{m} S\left(P_{k_{i}}\right) \subset S\left(P_{j}\right)
$$


This contradiction finishes the proof.

Remark 4.1. By slightly modifying the above argument, we can prove the following stronger result: If $S(P)$ is an algebraic surface and there exists a contractive affine map $f$ such that $S(P)$ contains the fixed point $z$ of $f$ and a non-periodic orbit $\left\{f^{n}(x)\right\}$ for some $x$, then $S(P)$ is unbounded. To see this, choose $k_{1}<\ldots<k_{m}$ so that each $P_{n}$ is a linear combination of the polynomials $P_{k_{1}}, \ldots, P_{k_{m}}$. If $S(P)$ is bounded, then we can pick $j$ large enough so that $\operatorname{diam}\left(S\left(P_{j}\right)\right)<\left|z-f^{k_{m}}(x)\right|$. This is a contradiction since $S\left(P_{j}\right) \supset \bigcap_{i=1}^{m} S\left(P_{k_{i}}\right) \supset\left\{z, f^{k_{m}}(x)\right\}$.

Example 4.2. It is clear that a hyperplane can contain a non-trivial self-affine set. In this example, we show that also other kinds of non-compact algebraic surfaces can have this property. Let $P: \mathbf{R}^{n} \rightarrow \mathbf{R}, P\left(x_{1}, \ldots, x_{n}\right)=x_{1}^{2}+\cdots+x_{n-1}^{2}-x_{n}$, and observe that, by Lemma 3.1, the parabola $\left\{\left(x_{1}, \ldots, x_{n}\right) \in \mathbf{R}^{n}: x_{n}=x_{1}^{2}\right.$ and $x_{2}=$ $\left.\cdots=x_{n-1}=0\right\} \subset S(P)$ contains non-trivial self-affine sets. It is also easy to see that $S(P)$ contains self-affine sets having dimension larger than one. Fix an interval $[a, b] \subset \mathbf{R}$ and define a mapping $\eta:[a, b]^{n-1} \rightarrow \mathbf{R}^{n}$ by setting $\eta\left(x_{1}, \ldots, x_{n-1}\right)=$ $\left(x_{1}, \ldots, x_{n-1}, x_{1}^{2}+\cdots+x_{n-1}^{2}\right)$. Let $\left\{c_{i}\left(x_{1}, \ldots, x_{n-1}\right)+\left(d_{i}, \ldots, d_{i}\right)\right\}_{i=1}^{\ell}$ be an affine IFS on $\mathbf{R}^{n-1}$ so that $[a, b]^{n-1}$ is the self-affine set generated by it. Define $f_{i}: \mathbf{R}^{n} \rightarrow \mathbf{R}^{n}$ by setting

$$
f_{i}\left(x_{1}, \ldots, x_{n}\right)=\left(\begin{array}{ccccc}
c_{i} & 0 & \cdots & 0 & 0 \\
0 & c_{i} & \cdots & 0 & 0 \\
\vdots & \vdots & \ddots & \vdots & \vdots \\
0 & 0 & \cdots & c_{i} & 0 \\
2 c_{i} d_{i} & 2 c_{i} d_{i} & \cdots & 2 c_{i} d_{i} & c_{i}^{2}
\end{array}\right)\left(\begin{array}{c}
x_{1} \\
x_{2} \\
\vdots \\
x_{n-1} \\
x_{n}
\end{array}\right)+\left(\begin{array}{c}
d_{i} \\
d_{i} \\
\vdots \\
d_{i} \\
(d-1) d_{i}^{2}
\end{array}\right)
$$

for all $\left(x_{1}, \ldots, x_{n}\right) \in \mathbf{R}^{n}$ and $i \in\{1, \ldots, \ell\}$. Since $f_{i}\left(\eta\left(x_{1}, \ldots, x_{n-1}\right)\right)=\eta\left(c_{i} x_{1}+\right.$ $\left.d_{i}, \ldots, c_{i} x_{d-1}+d_{i}\right)$ the image $\eta\left([a, b]^{n-1}\right) \subset S(P)$ is invariant under the affine IFS $\left\{f_{i}\right\}_{i=1}^{\ell}$.

We shall next introduce a general condition which guarantees the algebraic surface to contain self-affine sets. Suppose that $P: \mathbf{R}^{n} \rightarrow \mathbf{R}$ is a non-constant polynomial with real coefficients. We say that a contractive invertible affine map $f$ is a scaling factor for $P$ if there exists a constant $C \in \mathbf{R}$ such that

$$
P \circ f=C P \text {. }
$$

A polynomial $P$ is called self-affine if it has two scaling factors with distinct fixed points.

Example 4.3. Let $P: \mathbf{R}^{2} \rightarrow \mathbf{R}, P\left(x_{1}, x_{2}\right)=x_{2}-x_{1}$. It is easy to see that $f: \mathbf{R}^{2} \rightarrow \mathbf{R}^{2}, f\left(x_{1}, x_{2}\right)=\frac{1}{2}\left(x_{1}, x_{2}\right)$, and $g: \mathbf{R}^{2} \rightarrow \mathbf{R}^{2}, g\left(x_{1}, x_{2}\right)=\frac{1}{2}\left(x_{1}+1, x_{2}+1\right)$, are scaling factors for $P$ and have distinct fixed points.

The following proposition shows that a polynomial $P$ being self-affine is sufficient for the inclusion of self-affine sets.

Proposition 4.4. If $P: \mathbf{R}^{n} \rightarrow \mathbf{R}$ is a self-affine polynomial, then $S(P)$ contains a non-trivial self-affine set.

Proof. Let $f$ be a scaling factor for $P$ with a constant $C$. Note that there exists a non-singular $d \times d$ matrix $M$ with $\|M\|<1$ and $a \in \mathbf{R}^{n}$ so that $f(x)=M x+a$ 
for all $x \in \mathbf{R}^{n}$. Observe that

$$
f^{j}(x)=M^{j} x+\sum_{i=0}^{j-1} M^{i} a \rightarrow \sum_{i=0}^{\infty} M^{i} a=: x_{0}
$$

as $j \rightarrow \infty$, where $x_{0} \in \mathbf{R}^{n}$ is the fixed point of $f$. Choose $x \in \mathbf{R}^{n}$ such that

$$
\left|P\left(x_{0}\right)\right|+1<|P(x)| \text {. }
$$

Such a point $x$ exists since $P$ is not bounded. Since

$$
C^{j} P(x)=P \circ f^{j}(x) \rightarrow P\left(x_{0}\right)
$$

as $j \rightarrow \infty$ we may choose $j$ large enough so that $\left|C^{j} P(x)\right|<\left|P\left(x_{0}\right)\right|+1$. Thus $|C|<1$.

Let $h$ and $g$ be scaling factors for $P$ with distinct fixed points. If $f$ is any finite composition of the mappings $h$ and $g$, then $f$ is a scaling factor for $P$. If $C$ is the constant associated to the scaling factor $f$, then the above reasoning implies that $|C|<1$. Furthermore, if $x_{0}$ is the fixed point of $f$, then $P\left(x_{0}\right)=\operatorname{Pof}\left(x_{0}\right)=C P\left(x_{0}\right)$. Since $|C|<1$, this implies $P\left(x_{0}\right)=0$ and $x_{0} \in S(P)$. Recalling that $S(P)$ is closed it thus contains the self-affine set generated by the affine IFS $\{h, g\}$.

Remark 4.5. It would be interesting to characterize all the algebraic surfaces associated to self-affine polynomials. For example, in the two-dimensional case, is the surface always contained in a line through the origin? Of course, the ultimate open question here is to characterize all the algebraic surfaces containing self-affine sets.

Acknowledgement. Feng was partially supported by the HKRGC GRF grants (projects CUHK401013, CUHK14302415). The authors are grateful to Christoph Bandt for many valuable comments to improve the paper.

\section{References}

[1] Bandt, C., and A. Kravchenko: Differentiability of fractal curves. - Nonlinearity 24:10, 2011, 2717-2728.

[2] Falconer, K.: Fractal geometry. - John Wiley \& Sons Ltd., Chichester, 1990.

[3] Hutchinson, J. E.: Fractals and self-similarity. - Indiana Univ. Math. J. 30:5, 1981, 713-747.

[4] KÄENMÄKI, A.: On the geometric structure of the limit set of conformal iterated function systems. - Publ. Mat. 47:1, 2003, 133-141.

[5] KäenmäKI, A.: Geometric rigidity of a class of fractal sets. - Math. Nachr. 279:1, 2006, $179-187$.

[6] Mattila, P.: On the structure of self-similar fractals. - Ann. Acad. Sci. Fenn. Ser. A I Math. $7: 2,1982,189-195$.

[7] Mayer, V., and M. Urbański: Finer geometric rigidity of limit sets of conformal IFS. - Proc. Amer. Math. Soc. 131:12, 2003, 3695-3702 (electronic). 